\title{
The Tree Alternative Conjecture Under the Topological Minor Relation
}

\author{
Jorge Bruno \\ Department Digital Technologies \\ University of Winchester \\ Winchester, U.K. \\ jorge.bruno@winchester.ac.uk
}

\author{
Paul Szeptycki \\ Department of Mathematics and Statistics \\ York University \\ Toronto, ON, Canada \\ szeptyck@yorku.ca
}

Submitted: Jul 9, 2021; Accepted: Feb 3, 2022; Published: Feb 25, 2022

(C) The authors. Released under the CC BY-ND license (International 4.0).

\begin{abstract}
The Tree Alternative Conjecture concerns the sizes of equivalence classes of trees with respect to mutual embeddable relation. Indeed, it conjectures that the number of isomorphism classes of trees mutually embeddable with a given tree $T$ is either 1 or infinite - with instances of size $\aleph_{0}$ and $2^{\aleph_{0}}$. We prove its analogue within the family of locally finite trees with respect to the topological minor relation. More precisely, we prove that for any locally finite tree $T$ the size of its equivalence class with respect to the topological minor relation can only be either 1 or $2^{\aleph_{0}}$.
\end{abstract}

Mathematics Subject Classifications: 05C10, 05C63, 05C89

\section{Introduction and Background}

The well-known Tree Alternative Conjecture (TAC) [4] concerns the sizes of equivalence classes of trees with respect to mutual embeddable relation. Indeed, it states that the number of isomorphism classes of trees mutually embeddable with a given tree $T$ is either 1 or infinite. The conjecture is certainly true for finite trees and has been confirmed for a number of nontrivial classes of infinite trees. In [4] the authors prove the conjecture for all rayless trees, with instances of trees having equivalence classes of size $\aleph_{0}$ and $2^{\aleph_{0}}$, and in [3] the result is extended to rayless graphs. The author of [14] extends the result to include all rooted trees - where the size of the equivalence class of any rooted locally finite trees is just 1 - and a large collection of locally finite unrooted ones. And in [10] the conjecture is proven for so-called scattered trees.

We consider the analogous with respect to the topological minor relation $\leqslant$, where for trees $T, S$ we have $T \leqslant \sharp S$ if some subdivision of the tree $T$ is isomorphic to a subgraph 
of $S$. If $T \leqslant^{\sharp} S \leqslant^{\sharp} T$ then $S$ and $T$ are topologically equivalent, and we write $T \equiv{ }^{\sharp} S$ and denote by $[T]$ the equivalence class of $T$ under $\leqslant \sharp$. Trees that are topologically equivalent are said to be of the same topological type.

Conjecture 1. For any tree $T,[T]$ is either of size 1 or infinite.

The objective of this paper is to address Conjecture 1 with respect to the family of locally finite trees.

Theorem 2. For any locally finite tree $T$,

$$
|[T]| \in\left\{1,2^{\aleph_{0}}\right\} .
$$

In order to prove Theorem 2 we establish the result on the family of rooted locally finite trees. A rooted tree $(T, r)$ is one with a distinguished vertex $r$ called its root. Any rooted tree $(T, r)$ generates a partial ordering $\leqslant_{T}$ on its set of vertices by establishing that $s \leqslant_{T} t$ provided that the unique path from $r$ to $t$ contains $s$; if $s<t$ then we say that $s$ is below $t$ and that $t$ is above $s$. If no such path exists between vertices $s, t$ then we say they are incompatible. This is called the tree order on $(T, r)$. A tree $(T, r)$ is a rooted subtree of a tree $(S, s)$ if $T$ is a subtree of $S$ and $v \leqslant_{S} w \Longleftrightarrow v \leqslant_{T} w$ for all $v, w \in V(T)$. A subdivision of a tree $(T, r)$ is any rooted tree obtained by subdividing any number of its edges. An injective map $\phi: V(T) \rightarrow V(S)$ is a rooted minor embedding (or just embedding whenever its intended meaning is clear from context) if $\phi$ can be extended to an isomorphism between a subdivision of $(T, r)$ and the smallest rooted subtree $\left(S^{\prime}, s^{\prime}\right)$ of $(S, s)$ containing all vertices in $\phi(V(T)) ; \phi(r)=s^{\prime}$ and $s^{\prime}$ is the minimal element of $\phi(V(T))$ in $S$ with respect to the induced tree ordering on $(S, s)$. It follows that an embedding $\phi: V(T) \rightarrow V(S)$ preserves the tree order from $(T, r)$ : for $w, v \in V(T)$, $v \leqslant_{T} w$ if, and only if, $\phi(v) \leqslant_{S} \phi(w)$. If there exists an embedding $\phi: V(T) \rightarrow V(S)$ then we say that $(T, r)$ is a rooted topological minor of $(S, s)$ we write $(T, r) \leqslant(S, s)$. If $(T, r) \leqslant^{\sharp}(S, s)$ and $(T, r) \geqslant^{\sharp}(S, s)$ then we write $(T, r) \equiv^{\sharp}(S, s)$ and say that they are topologically equivalent. The equivalence class of a rooted tree $(T, r)$ under $\leqslant$ is denoted by $[(T, r)]$. As stated previously, we first establish the following theorem in order to prove Theorem 2.

Theorem 3. For any given rooted locally finite tree $(T, r)$,

$$
|[(T, r)]| \in\left\{1,2^{\aleph_{0}}\right\}
$$

A quasi-ordered set $(X, \leqslant)$ is well-quasi-ordered (wqo) if it is well-founded and all antichains are finite. The topological minor relation is a wqo on $\mathcal{T}$; Kruskal [8] established the well-quasi-ordering on all finite trees and Nash-Williams [13] proved the result for all infinite trees. In fact, Nash-Williams' proof relied on showing that the collection of all rooted infinite trees is wqo under the rooted topological minor relation as introduced above. We make use of the following equivalent characterisations for a quasi-ordered set $(X, \leqslant)$ to be a wqo [11]: 
- any sequence $x_{1}, x_{2}, \ldots$ in $X$ contains a pair $x_{i} \leqslant x_{j}$ with $i<j$; and

- any sequence $x_{1}, x_{2}, \ldots$ in $X$ has an increasing subsequence $x_{n_{1}} \leqslant x_{n_{2}} \leqslant \ldots$

We use some basic set-theoretic notation and terminology which can be found in [6] or [7]. Recall that $\aleph_{0}$ is the cardinality of the natural numbers and we use the settheoretic notation $\omega$ to denote the the set of natural numbers, $\mathbb{N}$, with 0 included. The continuum $2^{\aleph_{0}}$ denotes the cardinality of the set of real numbers (which is also equal to the cardinality of $\mathbb{N}^{\mathbb{N}}$, the set of all functions $f: \mathbb{N} \rightarrow \mathbb{N}$ ). Other than using $\leqslant \sharp$ for the topological minor relation, and $\equiv \sharp$ for the mutually topological minor equivalence relation, our graph-theoretic notation and terminology are standard and we refer to [5]. For example, $T \simeq S$ will be used to denote that $T$ and $S$ are isomorphic. In the what remains of this manuscript all trees are assumed to be locally finite.

\section{Rooted Trees}

For the following definitions we fix a rooted tree $(T, r)$ and, since there is no danger of ambiguity, we employ the term subtree to denote rooted subtree. For a vertex $v \in V(T, r)$ the splitting number of $v, \operatorname{sp}(v)$, is simply $\operatorname{deg}(v)-1$ when $v \neq r$ and $\operatorname{deg}(v)$ when $r=v$. Given a vertex $v \in V(T)$ the full subtree of $(T, r)$ rooted at $v,\left(T_{v}, v\right)$, is the subtree of $(T, r)$ induced by all vertices $w$ with $v \leqslant_{T} w$. The tree on $\omega$ rooted at 0 and determined by adding one edge between successive natural numbers $(n+1$ is connected to $n$ and $n+2$ by an edge) will be referred to as the ray. Any isomorphic copy of the ray, as a rooted subgraph of $(T, r)$, is also referred to as a ray. For a ray $R$ in $(T, r)$ we write $R_{n}$ to denote $R$ 's $n^{\text {th }}$ vertex along $T$ 's order. A ray $R$ in a rooted tree $(T, r)$ is principal if $r=R_{0}$. Given a vertex $v \in V(T, r)$ if $\left(T_{v}, v\right)$ is isomorphic to the ray then we say that $\left(T_{v}, v\right)$ is a simple ray. A simple ray is maximal if it is not a proper subtree of another simple ray in $(T, r)$. A path $p=\left(v_{0}, v_{1}, \ldots, v_{n}\right)$ in $(T, r)$ is a finite sequence of distinct vertices with $v_{i}$ adjacent to $v_{i-1}$. A path $p=\left(v_{0}, v_{1}, \ldots, v_{n}\right)$ is called a bare path if $s p\left(v_{i}\right)=1$ for all $0<i<n$. For convenience we write paths in ascending order; $v_{0}<_{T} v_{1}<_{T} \ldots<_{T} v_{n}$. The length of a path, $|p|$, is simply the number of vertices in it; the empty path has length 0 . A bare path is maximal if it is not a subgraph of a longer bare path. For $v$ a vertex of a rooted tree $(T, r)$, we define the level of $v$ to be $|p|-1$ where $p$ is the path connecting $r$ to $v$. We often employ the symbol $l_{T}(v)$ to denote this quantity. The root $r$ is the unique vertex with $l_{T}(r)=0$. It is immediate from the definition of $\leqslant^{\sharp}$ that given an embedding $\phi: V(T) \rightarrow V(S)$ witnessing $(T, r) \leqslant^{\sharp}(S, s)$ it must that that $l_{T}(v) \leqslant l_{S}(\phi(v))$ for all $v \in V(T)$.

For a tree $(T, r)$ we let $F_{T}=\{s \in V(T) \mid s p(s) \geqslant 2\}$ and say that $(T, r)$ is small if $F_{T}$ is finite. Notice that if $F_{T} \neq \varnothing$ then there is always exist a $v \in F_{T}$ with $v \leqslant_{T} w$ for all $w \in F_{T}$ (i.e., $v$ is the "first" vertex in $(T, r)$ with $s p(v)>1)$. We say that $(T, r)$ is self-similar if there exists a vertex $v>_{T} r$ and a subtree of $\left(T_{v}, v\right)$ of $(T, r)$ with $(T, r) \leqslant \sharp\left(T_{v}, v\right)$. It is well-known that two finite rooted trees are topologically equivalent if, and only if, they are isomorphic [8]. This is also true in the unrooted case. Our first objective is to extend this scenario to all small trees: two rooted small trees are topological minors of each other 
precisely when they are isomorphic. In order to do so we first show that not being small and having a self-similar full subtree - other than the ray - are equivalent notions.

Lemma 4. A tree $(T, r)$ is small if, and only if, no full subtree of $(T, r)$ - distinct from the ray - is self-similar.

Proof. $(\Rightarrow)$ Any subtree of a small tree is clearly small and, thus, we focus on proving that a small tree is itself not self-similar. Let $(T, r)$ be small - distinct from the ray and $\phi: V(T) \rightarrow V(T)$ be any embedding witnessing $(T, r) \leqslant \sharp(T, r)$. If $s \in F_{T}$ then $s p(\phi(s)) \geqslant 2$ and, by virtue of $F_{T}$ being finite, $\phi$ establishes a permutation on $F_{T}$. The topological minor relation is transitive and, as such, each $\phi^{k}: V(T) \rightarrow V(T), k \in \mathbb{N}$, is itself an embedding. Let $m \in \mathbb{N}$ be the smallest so that $\phi^{m}$ is the identity on $F_{T}$. This embedding $\phi^{m}$ then forces $s p(v)=s p(\phi(v))$ for all $v \in F_{T}$ since

$$
s p(v) \leqslant s p(\phi(v)) \leqslant \ldots \leqslant s p\left(\phi^{m}(v)\right)=s p(v) .
$$

Let $v \in F_{T}$ so that $v \leqslant_{T} s$ for all $s \in F_{T}$. Because $\phi$ preserves the order of $T$ and the preceding paragraph it follows that $\phi(v)=v$. Therefore, since $\phi$ is an embedding, $\phi(w)=w$ for all $w \leqslant_{T} v$. Since $r \leqslant_{T} v$ it follows that $\phi(r)=r$ and, hence, $(T, r)$ is not self-similar.

$(\Leftarrow)$ Let $(T, r)$ be any tree that is not small. It follows that $F_{T}$ is infinite and by König's lemma ([7] or [6]), there is a ray $R$ in $(T, r)$ with the property that for each $n \in \mathbb{N}$ there exists and $m \geqslant n$ with $R_{m} \in F_{T}$. To simplify notation, let $\left(v_{n}\right)$ denote the sequence of vertices of $R$ with $v_{n} \in F_{T}$. Since trees under the topological minor relation are wqo we can find a subsequence $\left(w_{k}\right)$ of $\left(v_{n}\right)$ with $\left(T_{w_{k}}, w_{k}\right) \leqslant\left(T_{w_{k+1}}, w_{k+1}\right)$ for all $k \in \mathbb{N}$. Hence, the full subtree of $(T, r)$ rooted at $w_{1}$ is self-similar.

Theorem 5. If $(T, r)$ is small then $|[(T, r)]|=1$.

Proof. We prove the claim by induction on the size of $F_{T}$. Let $(T, r)$ be small with $F_{T}=\varnothing$. It follows that $(T, r)$ is the ray or finite. In either case, if $(S, s) \equiv{ }^{\sharp}(T, r)$ for some rooted tree $(S, s)$ then clearly $(S, s) \simeq(T, r)$.

Let $k \in \mathbb{N}$ so that for any rooted tree $\left(T^{\prime}, r^{\prime}\right)$ with $\left|F_{T^{\prime}}\right| \leqslant k$ if $(S, s) \equiv^{\sharp}\left(T^{\prime}, r^{\prime}\right)$ for some rooted tree $(S, s)$ then $(S, s) \simeq\left(T^{\prime}, r^{\prime}\right)$. Next, let $(T, r)$ be any small tree with $\left|F_{T}\right|=k+1$, $(S, s)$ be any rooted tree topologically equivalent to $(T, r)$, and $\phi: V(T) \rightarrow V(S)$ witness $(T, r) \leqslant^{\sharp}(S, s)$ and $\psi: V(S) \rightarrow V(T)$ witness $(T, r) \geqslant \sharp(S, s)$. The embeddings $\phi$ and $\psi$ force $\left|F_{T}\right| \leqslant\left|F_{S}\right| \leqslant\left|F_{T}\right|$ and, therefore, $\left|F_{T}\right|=\left|F_{S}\right|$. Let $m \in \mathbb{N}$ be the smallest number for which $(\psi \circ \phi)^{m}$ is the identity on $F_{T}$ and $v \in F_{T}$ so that $v \leqslant_{T} u$ for all $u \in F_{T}$. Put $w=\phi(v)$. Since,

$$
s p(v) \leqslant s p(\phi(v)) \leqslant s p(\psi \circ \phi(v)) \leqslant \ldots \leqslant s p\left((\psi \circ \phi)^{m}(v)\right)=\operatorname{sp}(v)
$$

it follows that $s p(v)=s p(w)$ and $w \in F_{S}$. Put $N=s p(w)=s p(v)$. Since $\phi$ preserves the tree order of $(T, r)$ it follows that $u \geqslant_{S} w$ for all $u \in F_{S}$. In turn, the embedding $\phi$ maps the bare path $p: r, \ldots, v$ to the bare path $q: s, \ldots, w$, and the embedding $(\psi \circ \phi)^{m} \circ \psi$ maps the bare path $q$ to the bare path $p$. Hence, $\phi$ establishes a bijection between the 
bare paths $p$ and $q$ and $l_{T}(v)=l_{S}(w)$ (e.g., if $v=r$ then $w=s$ ). Next we show that $\phi$ witnesses $\left(T_{v}, v\right) \simeq\left(S_{w}, w\right)$.

Let $\left\{v_{i} \mid i \leqslant N\right\}$ and $\left\{w_{i} \mid i \leqslant N\right\}$ denote the collection of immediate successors of $v$ and $w$, respectively, and for each $i \leqslant N$ put $\left(T_{i}, v_{i}\right)$ and $\left(S_{i}, w_{i}\right)$ as the full subtrees of $(T, r)$ and $(S, s)$ rooted at $v_{i}$ and $w_{i}$, respectively. It follows that for each $i \leqslant N$, $\phi$ witnesses $\left(T_{i}, v_{i}\right) \leqslant^{\sharp}\left(S_{j_{i}}, w_{j_{i}}\right)$ for a unique $j_{i} \leqslant N$. Also, $(\psi \circ \phi)^{m} \circ \psi$ witnesses $\left(T_{i}, v_{i}\right) \geqslant \sharp\left(S_{j_{i}}, w_{j_{i}}\right)$ and since $\left|F_{T_{i}}\right| \leqslant k$ then $\left(T_{i}, v_{i}\right) \simeq\left(S_{j_{i}}, w_{j_{i}}\right)$, by the inductive hypothesis. It follows that $\left(T_{v}, v\right) \simeq\left(S_{w}, w\right)$, as claimed.

The objective in what remains of this section is to show that not being small implies an equivalence class of size $2^{\aleph_{0}}$.

Theorem 6. Let $(T, r)$ be distinct from the ray. If $(T, r)$ is self-similar then $|[(T, r)]|=$ $2^{\aleph_{0}}$.

Proof. Suppose that $(T, r)$ is self-similar. Let the embedding $\phi: V(T) \rightarrow V\left(T_{r_{n}}\right)$ witness $(T, r) \leqslant^{\sharp}(T, r)$ with $\phi(r)>_{T} r$. For each $n \in \mathbb{N}$, we then have $\phi^{n+1}(r)>_{T} \phi^{n}(r)$. Let $R$ be the unique principal ray in $(T, r)$ containing all $\phi^{n}(r)$; clearly, $\phi(V(R)) \subseteq V(R)$. Since $(T, r)$ itself is assumed to be different to the ray, it follows that infinitely many vertices in $R$ are of splitting number $\geqslant 2$, meaning that for each $n \in \mathbb{N}$ there exists $m>n$ with $s p\left(R_{m}\right) \geqslant 2$. Put $r_{0}=r$. Next, let $t_{1} \in \mathbb{N}$ be the smallest for which there exists a vertex $a \in V(R)$ succeeding $r_{0}$ with $\operatorname{sp}(a) \geqslant 2$ and $a<\phi^{t_{1}}(r)$. Put $r_{1}=\phi^{t_{1}}(r)$. Inductively define all other $r_{n}=\phi^{t_{n}}(r)$, where $t_{n} \in \mathbb{N}$ is the least such number for which there exists a vertex $a \in V(R)$ succeeding $r_{n-1}$ with $\operatorname{sp}(a) \geqslant 2$ and $a<\phi^{t_{n}}(r)$. Clearly, for each $n \in \mathbb{N}, r_{n}>_{T} r_{n-1}$ and the full subtree $\left(T_{r_{n}}, r_{n}\right)$ satisfies $\left(T_{r_{n}}, r_{n}\right) \equiv^{\sharp}(T, r)$ witnessed by the embedding $\phi^{t_{n}}: V(T) \rightarrow V(T)$. For each $n>0$ put $r_{n}^{\prime}$ as the unique vertex preceding $r_{n}$ and $e_{n}$ as the edge joining $r_{n}^{\prime}$ and $r_{n}$ as in Figure 1.

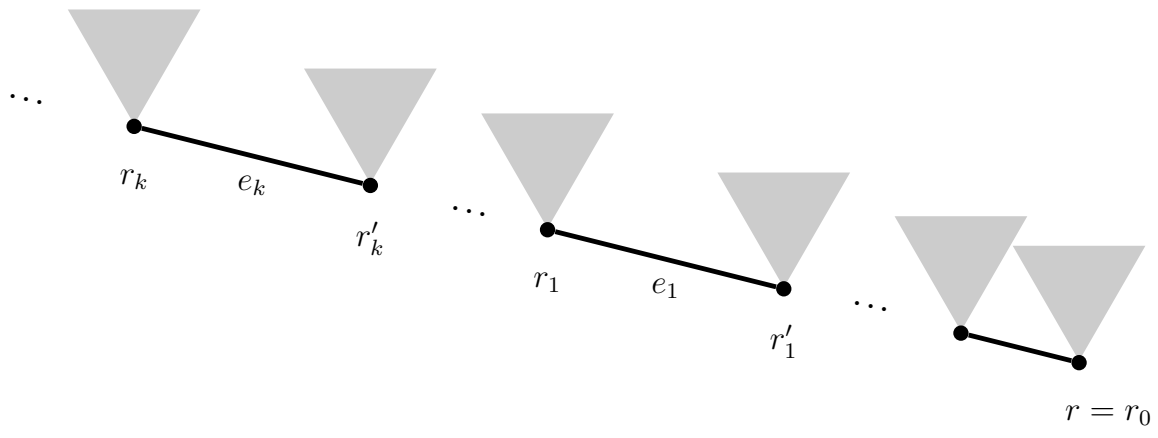

Figure 1: The tree $(T, r)$ with the ray $R$ highlighted.

For each $f: \mathbb{N} \rightarrow \mathbb{N}$ let $\left(T_{f}, r\right)$ denote the tree that results from the following subdivision of $(T, r)$ : for each $n \in \mathbb{N}$ subdivide $e_{n}$ into a bare path of length $f(n)$. Let $p_{n}$ denote the bare path of length $f(n)$ that replaces $e_{n}$ in $(T, r)$. Then for each $n \in \mathbb{N}$ let $r_{n, f}^{\prime}$ and $r_{n, f}$ denote the vertices in $\left(T_{f}, r\right)$ that are incident respectively with the initial 
and terminal vertices of $p_{n}$. These vertices correspond to the vertices $r_{n}^{\prime}$ and $r_{n}$ joined by $e_{n}$ in $(T, r)$. Note that since $s p\left(r_{n, f}\right)$ or $s p\left(r_{n, f}^{\prime}\right)$ may not equal 1 the bare path starting at $r_{n, f}^{\prime}$ and terminating at $r_{n, f}$, of length $f(n)+2$, may not be a maximal bare path.

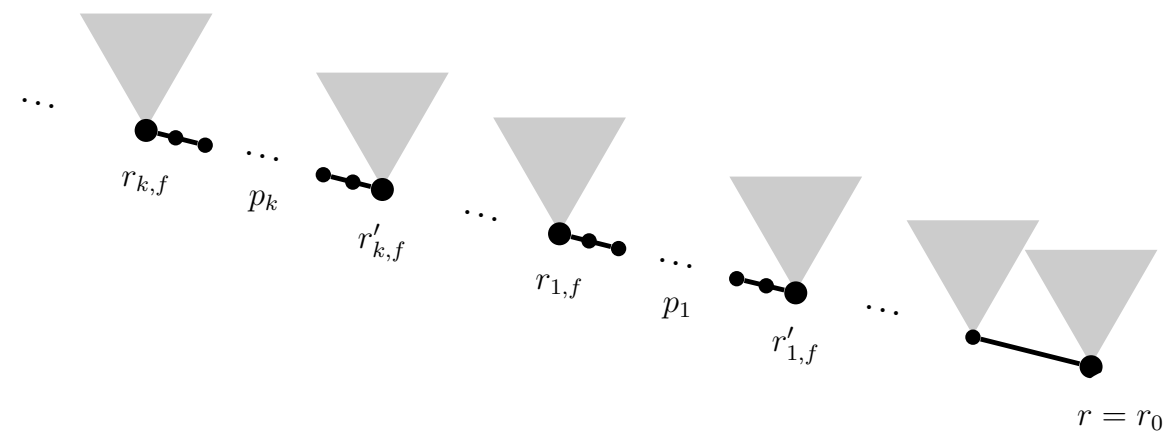

Figure 2: The tree $\left(T_{f}, r\right)$ with the ray $R$ highlighted.

Lemma 7. For any pair $f, g \in \mathbb{N}^{\mathbb{N}},\left(T_{f}, r\right) \equiv{ }^{\sharp}\left(T_{g}, r\right)$.

Proof. We show that for any $f$, we have that $\left(T_{f}, r\right) \equiv^{\sharp}(T, r)$. By transitivity of $\equiv^{\sharp}$, the lemma will follow. By design, $(T, r) \leqslant \sharp\left(T_{f}, r\right)$ for each $f$ since $\left(T_{f}, r\right)$ is a subdivision of $(T, r)$. We will establish the reverse inequality by recursively constructing a subdivision of $\left(T_{f}, r\right)$ and a rooted minor embedding of that subdivision into $(T, r)$ as follows.

By recursion on $k<\mathbb{N}$ we define subdivisions $p_{k}^{\prime}$ of $p_{k}$ and partial embeddings $\psi_{k}$ so that the following inductive hypotheses hold

(a) if $T_{f}^{k}$ is the tree obtained by applying all the subdivisions $\left\{p_{i}^{\prime}: i \leqslant k\right\}$ to $T_{f}$, then $\psi_{k}$ maps all vertices of $T_{f}^{k}$ not lying above $r_{k+1, f}^{\prime}$ to the subtree of $T$ consisting of all vertices of $T$ not lying above $v_{k+1}=\psi_{k}\left(r_{k+1, f}^{\prime}\right) \in R$.

(b) for $i<j \leqslant k$ we have that $\psi_{j}$ extends $\psi_{i}$.

Completing this recursive construction would complete the proof of Lemma 7 since the tree obtained from $T_{f}$ by applying all the subdivisions $\bigcup_{k \in \mathbb{N}} p_{k}^{\prime}$ and the embedding $\bigcup_{k \in \mathbb{N}} \psi_{k}$, would witness $\left(T_{f}, r\right) \leqslant \sharp(T, r)$.

To define $\psi_{1}$ and $p_{1}^{\prime}$, first map all vertices not lying above $r_{1, f}^{\prime}$ with the identity function (so the image is all vertices in $T$ not above $r_{1}^{\prime}$ ). By self-similarity, for all $n>1$ there exists an embedding $\psi$ mapping $\left(T_{r_{1}}, r_{1}\right)$ into $\left(T_{r_{n}}, r_{n}\right)$ with mapping $r_{1}$ to $r_{n}$ and $\psi(V(R)) \subseteq V(R)$ (one can calculate that $\psi=\phi^{t_{n}-t_{1}}$ in terms of our original embeddings used to define the $r_{n}$ 's). That $\psi(V(R)) \subseteq V(R)$ is important since it implies that the subtree $\left(S, r_{1}\right)$ generated by all vertices $v \geqslant_{T} r_{1}$ but $v \Varangle_{T} R_{m}$ for all $R_{m}>r_{1}$ is mapped to the subtree $\left(U, r_{n}\right)$ generated by all vertices $v \geqslant r_{n}$ but $v \ngtr R_{m}$ for all $R_{m}>r_{n}$.

Fix $n_{1} \in \mathbb{N}$ such that the length of the path from $r_{1}^{\prime}$ to $r_{n_{1}}$ is greater than or equal to $f(1)=\left|p_{1}\right|$. We may now subdivide an edge in $p_{1}$ to produce a path $p_{1}^{\prime}$ whose length is the same as the path from $r_{1}^{\prime}$ to $r_{n_{1}}$. Now extend our partial identity map by mapping $p_{1}^{\prime}$ 
isomorphically to that path (and so $r_{1, f}$ maps to $r_{n_{1}}$ ) and extending to the vertices lying above $r_{1, f}$ but not above $r_{2, f}^{\prime}$ using the embedding $\psi=\phi^{t_{n}-t_{1}}$ described above. Denote this partial embedding $\psi_{1}$ and note it maps $r_{2, f}^{\prime}$ onto some vertex $v_{2} \in V(R)$. Suppose we have defined subdivisions $p_{j}^{\prime}$ and partial embeddings $\psi_{j}$ for all $j<k$ satisifying (a) and (b) above.

We continue in the same manner, fixing a $n_{k}$ so that the length of the path from $v_{k}$ to $r_{n_{k}}$ is greater than or equal to $f(k)=\left|p_{k}\right|$. And we fix the embedding $\psi$ that embeds $\left(T_{r_{k}}, r_{k}\right)$ into $\left(T_{r_{n_{k}}}, r_{n_{k}}\right)$ with $\psi\left(r_{2}\right)=r_{n_{2}}$ ( $\psi$ can be calculated as a power of $\phi$ as above). We subdivide an edge of $p_{k}$ to obtain a $p_{k}^{\prime}$ of the same length as the path from $v_{k}$ to $r_{n_{k}}$, and extend our partial embedding $\psi_{k-1}$ by first mapping the the edge $p_{2}^{\prime}$ isomorphically onto the path from $v_{k}$ to $r_{n_{k}}$ (so that $r_{k, f}$ maps to $r_{n_{k}}$ ). And then we map all vertices lying above $r_{k, f}$ but not lying above $r_{k+1, f}^{\prime}$ using the embedding $\psi$. This defines $\psi_{k}$. By construction the subdivision $p_{k}^{\prime}$ and the partial embedding $\psi_{k}$ satisfy (a) and (b), completing the proof of Lemma 7.

While it is possible that distinct $g, f \in \mathbb{N}^{\mathbb{N}}$ generate isomorphic trees what we show next is that there are $2^{\aleph_{0}}$ many of them that are pairwise non-isomorphic. For each $n \in \mathbb{N}$ let $l_{n}$ denote collection of all vertices of $(T, r)$ that belong to the same level as $r_{n}^{\prime}$, the predecessor of $r_{n}$, and

$$
L_{n}=\left\{p: p \text { is a finite maximal bare path with initial vertex } p(0) \in l_{n}\right\} .
$$

It is worth highlighting that for a path $p$ to be in $L_{n}$ it must be that if $v$ is the terminal vertex of $p$ then $s p(v)>1$. Since between consecutive pairs $r_{n}, r_{n+1}$ there exist $v$ with $s p(v)>1$ we get that $L_{n} \neq \varnothing$. Since $(T, r)$ is locally finite, there are only finitely many finite maximal bare paths starting from a fixed vertex and so it follows that $M_{n}=$ $\max \left\{|p|: p \in L_{n}\right\}$ exists.

Let $f \in \mathbb{N}^{\mathbb{N}}$ so that $f(1)>M_{1}$. It follows that the tree $\left(T_{f}, r\right)$ fails to be isomorphic to $(T, r)$ since, by definition of $M_{1}$, there is no finite maximal bare path of length $\geqslant f(1)$ in $(T, r)$ starting at any point in the $l_{1}$, while, by design, there is such a bare path in $T_{f}$. This idea extends and we have the following

Lemma 8. Let $f, g \in \mathbb{N}^{\mathbb{N}}$ so that $f(n), g(n)>M_{n}$, for all $n \in \mathbb{N}$. Then $\left(T_{f}, r\right) \simeq\left(T_{g}, r\right)$ if, and only if, $f=g$.

Proof. Let $f, g \in \mathbb{N}^{\mathbb{N}}$ be as required. Put $G$ and $F$ as the unique principal rays in $\left(T_{g}, r\right)$ and $\left(T_{f}, r\right)$ that contain all $r_{n, g}$ and $r_{n, f}$, respectively; both subdivisions of $R$. Suppose that $\left(T_{g}, r\right)$ and $\left(T_{f}, r\right)$ are isomorphic and fix a bijection $b: V\left(T_{g}\right) \rightarrow V\left(T_{f}\right)$ witnessing $\left(T_{f}, r\right) \simeq\left(T_{g}, r\right)$.

We will prove that $b$ establishes an isomorphism between $G$ and $F$ and, therefore, that $f(n)=g(n)$ for all $n$. The proof is by induction on $n$ considering the restrictions of $b$ to $\left\{u \in V(G): u \leqslant r_{n, g}\right\}$.

First note that, as in the observation preceding the statement of the Lemma, $b$ must map $r_{1, g}^{\prime}$ to $r_{1, f}^{\prime}$ since all other vertices $v \in V\left(T_{f}\right)$ on the same level as $r_{1, f}^{\prime}$ precede either a ray or finite bare paths of length at most $M_{1}<g(1)$. Moreover, the subdivision of $e_{1}$ 
dictated by $g(1)$ above $r_{1, g}^{\prime}$ must be mapped into the subdivision of $e_{1}$ above $r_{1, f}^{\prime}$ dictated by $f(1)$. Again, this follows from the fact that $f(1), g(1)>M_{1}$. It follows that for any $G_{k}$ with $G_{k} \leqslant T_{g} r_{1, g}, b\left(G_{k}\right)=F_{k}$.

Let $u>_{T_{g}} r_{1, g}^{\prime}$ denote the first vertex above $r_{1, g}^{\prime}$ with splitting number at least 2, and observe that $u$ must lie in $G$, and so $u=G_{m_{1}}$ for some $m_{1} \in \mathbb{N}$. It follows that $r_{2, g}^{\prime} \geqslant_{T_{g}} G_{m_{1}} \geqslant_{T_{g}} r_{1, g}$ since all other vertices between $r_{1, g}^{\prime}$ and $r_{1, g}$ have splitting number 1. Since $b$ witnesses an isomorphism we have that $b\left(G_{m_{1}}\right)$ is the first vertex along $F$ with splitting number greater than 1 and so $b\left(G_{m_{1}}\right) \in F$ and so, $b\left(G_{k}\right)=F_{k}$ for all $k \leqslant m_{1}$. More importantly, since $G$ and $F$ are subdivisions of $R$ it must be that $G_{m_{1}}$ corresponds to the first vertex above $r_{1}$ with splitting number $\geqslant 2$. Whence, $b\left(r_{1, g}\right)=r_{1, f}$ and thus $f(1)=g(1)$.

Now assume that $n \in \mathbb{N}$ and that we have the following inductive hypotheses:

1. $b\left(G_{k}\right)=F_{k}$ for all $G_{k} \leqslant r_{n, g}$

2. $b\left(r_{i, g}\right)=r_{i, f}$ for all $i \leqslant n$

3. $f(i)=g(i)$ for all $i \leqslant n$.

By these assumptions we have that there is an $M$ such that $F_{M}=r_{n, f}$ and $G_{M}=r_{n, g}$ and $b$ maps $\left\{G_{i}: i \leqslant M\right\}$ isomorphically onto $\left\{F_{i}: i \leqslant M\right\}$.

Now, in the full subtree $\left(\left(T_{g}\right)_{r_{n, g}}, r_{n, g}\right)$ of $\left(T_{g}, r\right)$ let $l_{n+1}^{g}$ be the set of vertices in the same level as $r_{n+1, g}^{\prime}$ in $\left(\left(T_{g}\right)_{r_{n, g}}, r_{n, g}\right)$. Similarly let $l_{n+1}^{f}$ be the set of vertices in the same level as $r_{n+1, f}^{\prime}$ in the subtree $\left(\left(T_{f}\right)_{r_{n, f}}, r_{n, f}\right)$. By our inductive assumptions, we have that

$$
l_{n+1}^{g} \backslash\left\{r_{n+1, g}^{\prime}\right\}=l_{n+1}^{f} \backslash\left\{r_{n+1, f}^{\prime}\right\}=l_{n} \backslash\left\{r_{n+1}^{\prime}\right\} .
$$

Now the proof proceeds as in the base case: $b$ must map $r_{n+1, g}^{\prime}$ to $r_{n+1, f}^{\prime}$ since all other vertices $v \in l_{n+1}^{f}$ precede either a ray or a finite bare path of length at most $M_{n+1}<$ $g(n+1)$. Moreover, the subdivision of $e_{n+1}$ dictated by $g(n+1)$ above $r_{n+1, g}^{\prime}$ must be mapped into the subdivision of $e_{n+1}$ above $r_{n+1, f}^{\prime}$ dictated by $f(n+1)$. Again, this follows from the fact that $f(n+1), g(n+1)>M_{n+1}$. It follows that for any $G_{k}$ with $G_{k} \leqslant T_{g} r_{n+1, g}$, we have $b\left(G_{k}\right)=F_{k}$.

Let $G_{m_{n+1}}>_{T_{g}} r_{n+1, g}^{\prime}$ denote the first vertex along $G$ with splitting number at least 2 (that it lies in $G$ is reasoned as above). It follows that $r_{n+2, g}^{\prime} \geqslant_{T_{g}} G_{m_{n+1}} \geqslant_{T_{g}} r_{n+1, g}$ since all other vertices between $r_{n+1, g}^{\prime}$ and $r_{n+1, g}$ have splitting number 1 . Since $b$ witnesses an isomorphism we have that $b\left(G_{m_{n+1}}\right)=F_{m_{n+1}}$ and that $F_{m_{n+1}}$ is the first vertex along $F$ with splitting number greater than 1 . Therefore, $b\left(G_{k}\right)=F_{k}$ for all $k \leqslant m_{n+1}$. More importantly, since $G$ and $F$ are subdivisions of $R$ it must be that $G_{m_{n+1}}$ corresponds to the first vertex above $r_{n+1}$ with splitting number $\geqslant 2$. Whence, $b\left(r_{n+1, g}\right)=r_{n+1, f}$ and thus $f(n+1)=g(n+1)$. And the other inductive hypothesis for $n+1$ are verified. This completes the proof of the Lemma.

Of course, there are $2^{\aleph_{0}}$ many $f \in \mathbb{N}^{\mathbb{N}}$ with $f(n)>M_{n}$, for all $n \in \mathbb{N}$ completing the proof of the Theorem. 
We are now ready to prove Theorem 3 by extending the proof of Theorem 6 to all rooted trees that are not small.

Theorem 3. For any given rooted locally finite tree $(T, r)$,

$$
|[(T, r)]| \in\left\{1,2^{\aleph_{0}}\right\} .
$$

Proof. In view of the last two results, we need only show that any rooted tree $(T, r)$ with a self-similar full subtree is topologically equivalent to continuum many non-isomorphic trees. Let $(T, r)$ have a self-similar full subtree $\left(T_{v}, v\right)$ with $v>r$ (the case where $r=v$ was dealt with in the previous theorem). By Theorem 3 we have that $\left|\left[\left(T_{v}, v\right)\right]\right|=2^{\aleph_{0}}$. Let

$$
\mathcal{A}=\left\{\left(S_{\kappa}, s_{\kappa}\right) \mid \kappa<2^{\aleph_{0}}\right\}
$$

be a collection of continuum many pairwise non-isomorphic rooted trees topologically equivalent to $\left(T_{v}, v\right)$. Let $w$ denote the predecessor of $v$ and set

$$
M=\max \left\{\left|p: x_{1}, \ldots, x_{n}\right|: p \text { is bare and } l_{T}\left(x_{1}\right)=l_{T}(w)\right\} .
$$

For each $\kappa<2^{\aleph_{0}}$ let $\left(T_{\kappa}, r\right)$ denote the tree generated from $(T, r)$ replacing $\left(T_{v}, v\right)$ with $\left(S_{k}, s_{k}\right)$ and by subdividing the edge joining $v$ and $w$ with a bare path of length $M+1$. By the same arguments employed in Theorem 3 it follows that $(T, r) \equiv{ }^{\sharp}\left(S_{\kappa}, s_{\kappa}\right)$ for all such $\kappa$. Next we show that no pair of trees from $\{(T, r)\} \cup \mathcal{A}$ are isomorphic.

By design, for any $\kappa<2^{\aleph_{0}}, l_{T}(w)=d_{T_{\kappa}}(w)$ and all $\left(T_{\kappa}, r\right)$ have a bare path of length $M+1$ starting at the level of $w$. All bare paths in $(T, r)$ starting at the level of $w$ have maximum length of $M$. This then demonstrates that $\left(T_{\kappa}, r\right) \not \equiv \#(T, r)$ for all $\kappa<2^{\aleph_{0}}$. Finally, assume there exists a bijection $b: V\left(T_{\gamma}\right) \rightarrow V\left(T_{\kappa}\right), \kappa<\gamma<2^{\aleph_{0}}$, witnessing $\left(T_{\gamma}, r\right) \simeq\left(T_{\kappa}, r\right)$. Put $p_{\gamma}: w, \ldots, s_{\gamma}$ and $p_{\kappa}: w, \ldots, s_{\kappa}$ as the bare paths of length $M+1$ starting at $w$. Any other bare path $p: x_{1}, \ldots, x_{n}$ in either tree with $x_{1}$ at the same level as $w$ must have $|p| \leqslant M$. This then justifies that $b(w)=w$. Since both paths $p_{\kappa}$ and $p_{\gamma}$ are bare and the bijection $b$ witnesses $\left(T_{\gamma}, r\right) \simeq\left(T_{\kappa}, r\right)$ then $b\left(s_{\gamma}\right)=s_{\kappa}$. Recall that the end vertices $s_{\gamma}$ and $s_{\kappa}$ of $p_{\gamma}$ and $p_{\kappa}$ denote the roots of the full subtrees $\left(S_{\gamma}, s_{\gamma}\right)$ and $\left(S_{\kappa}, s_{\kappa}\right)$ of $\left(T_{\gamma}, r\right)$ and $\left(T_{\kappa}, r\right)$, respectively. In turn, the bijection $b$ restricted to the full subtree $\left(S_{\gamma}, s_{\gamma}\right)$ must witness $\left(S_{\gamma}, s_{\gamma}\right) \simeq\left(S_{\kappa}, s_{\kappa}\right)$. A contradiction.

\section{Unrooted trees}

The results obtained in the previous section can be extended to the unrooted case. The notion of being small and Lemma 4 are almost identical; an unrooted tree $T$ is small if the set $F_{T}=\{v \in V(T) \mid \operatorname{deg}(v) \geqslant 3\}$ is finite. The ray is the tree on $\omega$ determined by adding one edge between successive natural numbers without reference to a root. The double ray is the tree on $\mathbb{Z}$ by adding one edge between successive integers.

Theorem 2. For any locally finite tree $T$,

$$
|[T]| \in\left\{1,2^{\aleph_{0}}\right\} .
$$


Proof. Let $T$ be small and let $S$ be any other tree with $S \equiv^{\sharp} T$. If $\left|F_{T}\right|=0$ then either $T$ is finite, the ray or the double ray. In any of those scenarios the result follows immediately. Let $T$ with $\left|F_{T}\right|>0$ and $S \simeq T$ with $\phi: V(S) \rightarrow V(T)$ and $\psi: V(T) \rightarrow V(S)$ witnessing, $S \leqslant \sharp T$ and $T \leqslant \sharp S$, respectively. As with Theorem 5 , put $m \in \mathbb{N}$ as the smallest number with $(\psi \circ \phi)^{m}$ as the identity on $F_{T}$. Choose any $v \in F_{T}$ and put $w=\phi(v)$. Consider the trees $T$ and $S$ as rooted trees $(T, v)$ and $(S, w)$. The embedding $\phi$ witnesses $(T, v) \leqslant \sharp(S, w)$ and $(\psi \circ \phi)^{m-1} \circ \psi: V(S) \rightarrow V(T)$ witnesses $(T, v) \geqslant \sharp(S, w)$. By Theorem 5 the result follows.

To finish this theorem, let $T$ be any unrooted tree that is not small and choose any $r \in V(T)$. From Theorem 3 we are guaranteed $2^{\aleph_{0}}$ many $f, g \in \mathbb{N}^{\mathbb{N}}$ with $\left(T_{f}, r\right) \equiv{ }^{\sharp}\left(T_{g}, r\right)$ and $\left(T_{f}, r\right) \not\left(T_{g}, r\right)$; all topologically equivalent to $(T, r)$ but non-isomorphic to $(T, r)$. Denote the collection of all such trees as $A(T)$ and consider each $\left(T_{f}, r\right)$ as an unrooted tree; clearly $T \equiv \sharp T_{f}$ for all $\left(T_{f}, r\right) \in A(T)$. If $T \simeq T_{f}$ for some $\left(T_{f}, r\right) \in A(T)$ then there must exist a vertex $r_{f} \in V(T) \backslash\{r\}$ with $\left(T, r_{f}\right) \simeq\left(T_{f}, r\right)$; otherwise, if $r_{f}=r$ then $\left(T_{f}, r\right) \simeq(T, r)$. Any other $\left(T_{g}, r\right) \in A(T)$ with $T \simeq T_{g}$ must be guaranteed a different $r_{g} \in V(T) \backslash\left\{r, r_{f}\right\}$ with $\left(T, r_{g}\right) \simeq\left(T_{g}, r\right)$; otherwise $\left(T_{g}, r\right) \simeq\left(T_{f}, f\right)$. There are countably many vertices in $T$ and $2^{\aleph_{0}}$ many elements in $A(T)$. Hence, continuum many $T_{f}$ are non-isomorphic to $T$. This same argument applies to all $\left(T_{f}, r\right) \in A(T)$. Hence, all $2^{\aleph_{0}}$ unrooted versions of trees from $A(T)$ can be isomorphic to, at most, countably many other unrooted $T_{f}$, yielding $|[T]|=2^{\aleph_{0}}$.

\section{Strong Topological Minor}

One could argue that the true analogue of TAC for rooted trees under the topological minor relation would require that an embedding $\phi: V(T) \rightarrow V(S)$ witnessing $(T, r) \leqslant \sharp$ $(S, s)$ should map $\phi(r)=s$. This stronger notion is not standard and certainly not the one originally employed by Kurskal and Nash-Williams; the overriding reason being that, of course, this extra requirement is not necessary to extend the well-quasi-ordering of rooted trees over to the unrooted case. Similarly, our proof of Theorem 2 does not require this additional condition. In spite of this we think it is important to remark that Theorem 3 and its corollary apply in spades to this more restrictive scenario.

Momentarily define injective an map $\phi: V(T, r) \rightarrow V(S, s)$ to be a strong embedding if $\phi$ can be extended to an isomorphism between a subdivision of $(T, r)$ and the smallest rooted subtree $\left(S^{\prime}, s^{\prime}\right)$ of $(S, s)$ containing all vertices in $\phi(V(T, r))$ and $\phi(r)=s$. In which case, we say that $(T, r)$ is a strong topological minor of $(S, s)$ and write $(T, r) \leqslant(S, s)$. If $(T, r) \leqslant^{*}(S, s)$ and $(T, r) *^{*}(S, s)$ then $(T, r) \equiv^{*}(S, s)$, and we write $[(T, r)]^{*}$ to denote the equivalence class of $(T, r)$ under $\leqslant^{*}$. The proof of Theorem 3 can be modified to prove the following result.

Theorem 9. For a given rooted locally finite tree $(T, r)$,

$$
\left|[(T, r)]^{*}\right| \in\left\{1,2^{\aleph_{0}}\right\} .
$$

Proof. Clearly, $|[(T, r)]| \geqslant|[(T, r)]|^{*}$ and, therefore, if $(T, r)$ is small then it remains true that $\left|[(T, r)]^{*}\right|=1$. If $(T, r)$ is not small then we must only notice that the proof 
of Theorem 2 generates a collection of continuum many pairwise non-isomorphic trees $\left\{\left(T_{\gamma}, r\right) \mid \gamma<2^{\aleph_{0}}\right\}$ all topologically equivalent to $(T, r)$. Moreover, the embeddings $\phi_{\gamma}$ : $V(T) \rightarrow V\left(T_{\gamma}\right)$ and $\psi_{\gamma}: V\left(T_{\gamma}\right) \rightarrow V(T)$ witnessing $\left(T_{\gamma}, r\right) \leqslant \sharp(T, r)$ and $\left(T_{\gamma}, r\right) \geqslant \sharp(T, r)$ map $r$ to $r$.

\section{Concluding Remarks}

Conjecture 1, in its full generality, remains opens. Given trees $T$ and $S$ put $T \preceq S$ whenever $T$ embeds in $S$, and $T \sim S$ when both embed into each other. The Tree Alternative Conjecture then states that the equivalence class of a tree $T$ with respect to $\sim,[T]^{\sim}$, is either infinite or of size 1. Mutual embeddability is obviously stronger than topological equivalence: $\left|[T]^{\sim}\right| \leqslant|[T]|$ for any tree $T$. In fact, when $T$ is locally finite and rooted it is straightforward to show $[14]$ that $\left|[T]^{\sim}\right|=1$. Whereas in the topological minor case, $|[T]|=1$ only for small trees. In view of the above and since TAC has been solved for all rooted trees by Tyomkyn in [14], Conjecture 1 with respect to rooted trees can be reduced to trees $(T, r)$ with $\left|[(T, r)]^{\sim}\right|=1$.

Question 10. Given a rooted tree $(T, r)$ with $\left|[(T, r)]^{\sim}\right|=1$ is $[T]$ either infinite or of size 1.

It is interesting to notice that the 1 or $2^{\aleph_{0}}$ complementary scenario witnessed with locally finite tree under the topological minor relation does not extend to all trees. Consider the following rooted trees, as illustrated in Figure 3 (Fig. 1 from [4]), with topological equivalence classes of size $\aleph_{0}$ and $2^{\aleph_{0}}$, respectively. The first tree $T_{1}$ can be described as having a distinguished vertex to which countably many paths of length 2 are attached. For each $n$, the tree with $n$ paths of length 1 and countably many paths of length 2 attached to a single vertex is topologically equivalent but not isomorphic. The class of all trees topologically equivalent includes these plus one more, the tree with countably many many paths of length 1 and countably many paths of length 2 attached to a single vertex. The second tree $T_{2}$ can be described as having a distinguished vertex to which a path of length $n$ is attached for each $n$. This tree has $2^{\aleph_{0}}$ many topologically equivalent and pairwise non-isomorphic trees. Indeed, one corresponding to each possible subset of $\mathbb{N}$.

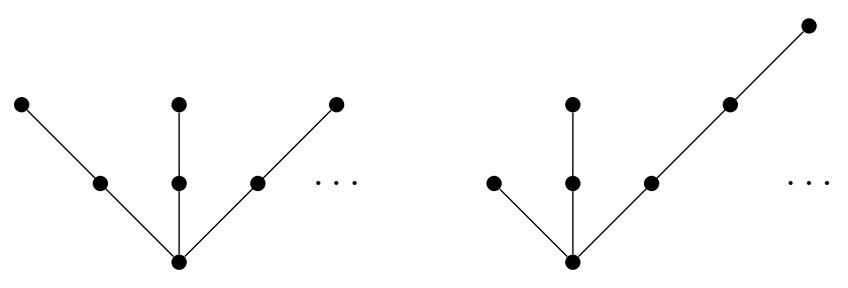

Figure 3: Trees $T_{1}$ and $T_{2}$.

We expect that the only possible cardinalities for the size of equivalence classes of locally countable trees under topological equivalence are $1, \aleph_{0}$ and $2^{\aleph_{0}}$. And assuming that TAC is true, we conjecture the same for the mutual embeddibility relation $\sim$. 
An affine topic to the size of equivalence classes of trees under the topological minor relation is that of the number of equivalence classes. Matthiesen [12] provided an existential proof that the number $\lambda$ of topological types of rooted locally finite tree must be uncountable and a constructive argument can be found in [1]. Since $\aleph_{1} \leqslant \lambda \leqslant \mathfrak{c}$, finding the exact value of $\lambda$ becomes non-trivial in the absence of the Continuum Hypothesis. In [2] it is shown that $\lambda=\omega_{1}$ for the collection of all rooted locally finite trees with countable many rays. The following question remains open.

Question 11. Is the number of equivalence classes of rooted locally finite trees under the topological minor relation $\omega_{1}$ ?

\section{Acknowledgements}

We would like to express our sincere gratitude to the referees for their insightful and very useful comments.

\section{References}

[1] Bruno, J. A family of $\omega_{1}$ topological types of locally finite trees, Discrete Mathematics, 340:794-795, 2017.

[2] Bruno, J. and Szeptycki, P. There are exactly $\omega_{1}$ topological types of locally finite trees with countably many rays, Fundamenta Mathematicae, 256: 243-259, 2022.

[3] Bonato, A., Bruhn, H., Diestel, R., Sprüssel, P., Twins of rayless graphs J. Combin. Theory Ser. B, 101: 60-65, 2011.

[4] Bonato, A. and Tardif, C., Mutually embeddable graphs and the tree alternative conjecture, Journal of Combinatorial Theory, Series B, 96:874-880, 2006.

[5] Diestel, R., Graph Theory, Graduate Texts in Mathematics, 2010.

[6] Just, W. and Weese, M, Discovering Modern Set Theory, I, American Mathematical Society, 1996.

[7] Kunen, K., Set Theory, College Publications, 2011.

[8] Kruskal, J. B. Well-quasi-ordering, the tree theorem, and Vazsonyi's conjecture, Transactions of the American Mathematical Society, 95 (2): 210-225, 1960.

[9] Kurskal, J. B., The Theory of Well-quasi-orderings: a frequently discovered concept, Journal of Combinatorial Theory, Series A, 13:297-305, 1972.

[10] C. Laflamme, M. Pouzet, and N. Sauer, Invariant subsets of scattered trees. An application to the tree alternative property of Bonato and Tardif, Abh. Math. Sem. Univ. Hamburg 87:369-408, 2017.

[11] Milner E.C. Basic WQO- and BQO-Theory. Graphs and Order. Springer, 1985.

[12] Matthiesen, L. There are uncountably many topological types of locally finite trees Journal of Combinatorial Theory, Series B, 96(5):758-760, 2006. 
[13] Nash-Williams, C. A., On well-quasi-ordering infinite trees, Proc. Cambridge Philos. Soc. v61 (1965), 697-720.

[14] Tyomkyn, M., A proof of the rooted tree alternative conjecture, Discrete Mathematics, 309, pp. 5963-5967, 2008. 